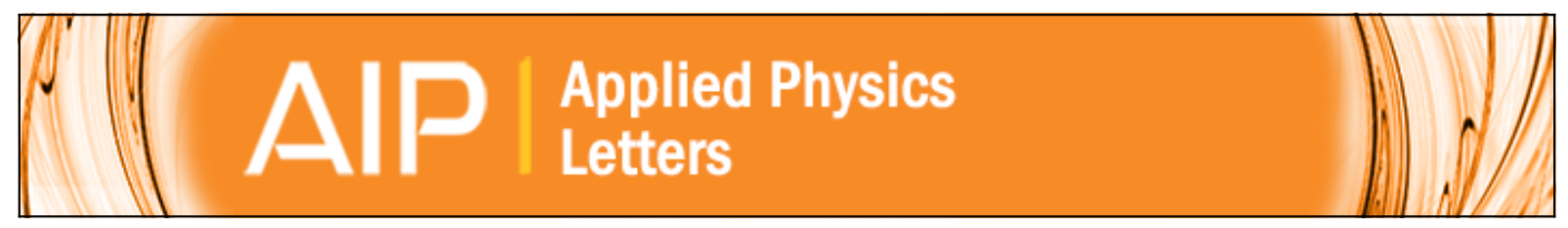

\title{
Weak localization in ultrananocrystalline diamond
}

J. J. Mareš, P. Hubík, J. Krištofik, D. Kindl, M. Fanta, M. Nesládek, O. Williams, and D. M. Gruen

Citation: Applied Physics Letters 88, 092107 (2006); doi: 10.1063/1.2176853

View online: http://dx.doi.org/10.1063/1.2176853

View Table of Contents: http://scitation.aip.org/content/aip/journal/apl/88/9?ver=pdfcov

Published by the AIP Publishing

\section{AlP Re-register for Table of Content Alerts}

\section{Create a profile. \\ Sign up today!}




\title{
Weak localization in ultrananocrystalline diamond
}

\author{
J. J. Mareš, ${ }^{a)}$ P. Hubík, J. Krištofik, D. Kindl, and M. Fanta \\ Institute of Physics, Academy of Sciences of the Czech Republic, Cukrovarnická 10, 16253 Prague 6, \\ Czech Republic \\ M. Nesládek \\ CEA-LIST (Recherche Technologique), CEA-Saclay, 91191 Gif sur Yvette, France \\ O. Williams \\ Center for Nanoscale Materials, Argonne National Laboratory, Argonne, Illinois 60439 \\ D. M. Gruen \\ Materials Science Division, Argonne National Laboratory, Argonne, Illinois 60439
}

(Received 31 August 2005; accepted 4 January 2006; published online 2 March 2006)

In this letter we present results of magnetotransport measurements, carried out on heavily nitrogen-doped ultrananocrystalline diamond films, prepared by plasma-enhanced chemical vapor deposition. This material having at room temperature appreciably high electric conductivity $\left(\sim 4200 \mathrm{~S} \mathrm{~m}^{-1}\right)$ revealed surprisingly at Kelvin temperatures a giant negative magnetoresistance reaching up to $22 \%$ at $1.28 \mathrm{~K}$ and at $8 \mathrm{~T}$. The analysis of experimental data has borne evidence of the fact that the transport in this subsystem has a character of low-dimensional disordered metal and it is controlled by quantum interference effects of electrons resulting in their weak localization. (C) 2006 American Institute of Physics. [DOI: 10.1063/1.2176853]

Microwave plasma enhanced chemical vapor deposition (PECVD) grown ultrananocrystalline diamond (UNCD) thin films ${ }^{1-3}$ are a new promising derivation of synthetic-CVD diamond, interesting for applications in electronics and especially bioelectronics. ${ }^{4}$ In contrast to single-crystalline diamond, a wide band gap semiconductor which is refractory with respect to the effective and sufficiently shallow $n$-type doping, ${ }^{5}$ UNCD can be doped relatively easily to an appreciable level simply by adding nitrogen during the PECVD film growth. For example, by means of an admixture of $20 \%$ of nitrogen $\left(\mathrm{N}_{2}\right)$ into the working gas, astonishingly high electric conductivity $\left(\sim 1.4 \times 10^{4} \mathrm{~S} \mathrm{~m}^{-1}\right)$ of the resulting UNCD layer may be achieved. ${ }^{2}$ The segregation of nitrogenrich component at the grain boundaries during the growth has been accounted for as a main reason for establishment of a topologically rather complicated, highly doped conductive subsystem. On the basis of the evaluation of the temperature dependences of the conductivity ${ }^{3,6,7}$ the mechanism controlling the electron transport has been studied with several suggestions for its explanation, including a hopping via strongly localized states. The unresolved questions concerning the somewhat atypical transport behavior of UNCD, together with the fact that some diamond materials may even reveal superconductivity, provide, as we believe, sufficient motivation for a systematic research into the low-temperature (i.e., quantum) phenomena in these materials.

Nitrogen doped UNCD thin films of grain size $\sim 16 \mathrm{~nm}$ were grown ${ }^{3}$ by microwave PECVD technique from gas phase containing $79 \% \mathrm{Ar}, 1 \% \mathrm{CH}_{4}$, and $20 \% \mathrm{~N}_{2}$ (total gas pressure $\sim 1.3 \times 10^{4} \mathrm{~Pa}$, microwave power $\sim 750 \mathrm{~W}$, substrate temperature $\sim 800{ }^{\circ} \mathrm{C}$ ). The films of thickness of $\sim 0.8 \mu \mathrm{m}$ were deposited onto standard highly insulating quartz substrates, enabling reliable transport measurements. The samples were then cut in approximately $5 \mathrm{~mm} \times 5 \mathrm{~mm}$

${ }^{a)}$ Electronic mail: semicon@fzu.cz squares and at the corners provided with vacuum evaporated $\mathrm{Ti} / \mathrm{Au}$ based contacts. For the magnetoresistance measurements within the range of $1.2-4.2 \mathrm{~K}$ and at fields up to $8 \mathrm{~T}$, we used the four-point van der Pauw technique, employing an Oxford Instruments AVS-47 resistance bridge as a measuring device.

Prior to the low temperature magnetotransport measurements, which were the main subject of this work, standard tests at temperatures between 90 and $440 \mathrm{~K}$ had been performed. In this temperature range, and at magnetic fields up to $0.4 \mathrm{~T}$, practically no traces of magnetoresistance effects were found and only a very small Hall coefficient (corresponding to an electron mobility of $\sim 2 \times 10^{-4} \mathrm{~m}^{2} / \mathrm{V} \mathrm{s}$ ) was observed. On the other side, the samples revealed a remarkably high, weakly temperature dependent electrical conductivity. Such typical temperature dependence of the electrical conductivity plotted in Arrhenius coordinates is depicted in Fig. 1. When approaching lower temperatures, the slope of the curve corresponding to the apparent activation (ionization) energy $E_{A}$ for the carriers involved continuously diminishes. The small and varying value of $E_{A}$, which is everywhere $<k T$, cannot, as we claim, represent an ordinary activated process but it is more reminiscent of a special kind of disordered metal. Indeed, such a view may be supported by an estimate of Mott's metallic transition threshold $\sigma_{M}$ made for this particular case. The relevant formula reads ${ }^{8}$

$$
\sigma_{M}=\left(8 \pi \kappa \varepsilon_{0} / \hbar\right) \varepsilon E_{A},
$$

where the relative permittivity $\varepsilon=5.5$ and the dimensionless purely geometrical constant $\kappa \approx 0.03$. If we now identify tentatively electron ionization energy $E_{A}$ with the directly observed slope of temperature dependence plotted in Arrhenius coordinates, reasonable limits for $\sigma_{M}$ may be obtained. At RT, for example, where $E_{A}(\mathrm{RT}) \approx 9 \mathrm{meV}$, one obtains from formula (1) for Mott's threshold an estimate $\sigma_{M}(\mathrm{RT})$ $\approx 500 \mathrm{~S} \mathrm{~m}^{-1}$. Since the experimental value of conductivity at this point is $\sim 4200 \mathrm{~S} \mathrm{~m}^{-1}>\sigma_{M}(\mathrm{RT})$ and as the slope of the 


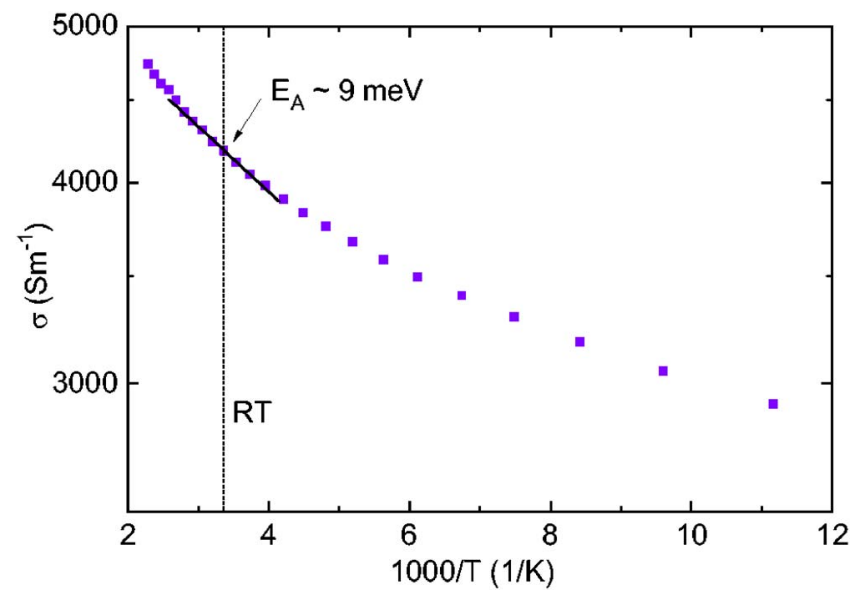

FIG. 1. (Color online) Arrhenius plot of temperature dependence of conductivity of nitrogen-doped UNCD sample $\left(20 \%\right.$ of $\mathrm{N}_{2}$ in working gas, thickness of diamond film $\sim 0.8 \mu \mathrm{m}$ ). The slope of the curve corresponding to the room temperature value of the apparent activation energy $E_{A}(\mathrm{RT})$ $\sim 9 \mathrm{meV}$ is shown explicitly.

experimental curve which is proportional to $\sigma_{M}$ decreases, approaching low temperatures, more progressively than the absolute value of conductivity $\sigma$, it can be suggested that the conductive subsystem has very likely a character of Mott's metal consisting of a band of extended states at the Fermi level.

Figure 2 depicts a set of magnetoresistance curves measured at magnetic fields perpendicular to the sample and at various temperatures ranging from 1.28 to $3.8 \mathrm{~K}$. At zero magnetic field the resistance increases as the temperature decreases, the fact which may be interpreted as a symptom of electron localization. For magnetic fields in close vicinity to zero there is only a small variation of the sample resistance with increasing magnetic field. After passing a certain point $B_{k}$, however, the dependences start quickly to decrease. The negative slope of these curves depends on temperature and is appreciably steeper at lower temperatures. The total decrease of resistance due to a magnetic field of $8 \mathrm{~T}$ is remarkably large, reaching a value of $22 \%$ at $\sim 1.28 \mathrm{~K}$. As far as we know, the only effect which can account for such a huge (giant) negative magnetoresistance (NMR) and other features observed in our experiment is the effect of weak localization. The essentials of this effect are the following.

In the case where the plane wave representing an electron in the (semi)conductor meets a cluster of scattering centers, it splits into a system of partial waves. Under favorable conditions these partial waves can add constructively, giving rise to a relatively stable stationary interference pattern. The existence of a stationary pattern means, however, that the electron is effectively localized in the vicinity of the cluster. This type of localization, playing an extraordinary role in the interpretation of low-temperature transport data of disordered metals and semiconductors, is known in the literature as a weak localization. ${ }^{9}$ For the realization of an interference process leading to the weak localization, the wavelength and phase coherence of all partial waves involved have to be preserved. In order to ensure these conditions, all the scattering events must be elastic and the electron wave phase coherence length $L_{\varphi}$ must exceed the characteristic extension of the scattering cluster. Since the interference is, as a rule, very sensitive to all types of perturbations, it will distinctly depend also on the presence of external electromagnetic fields.

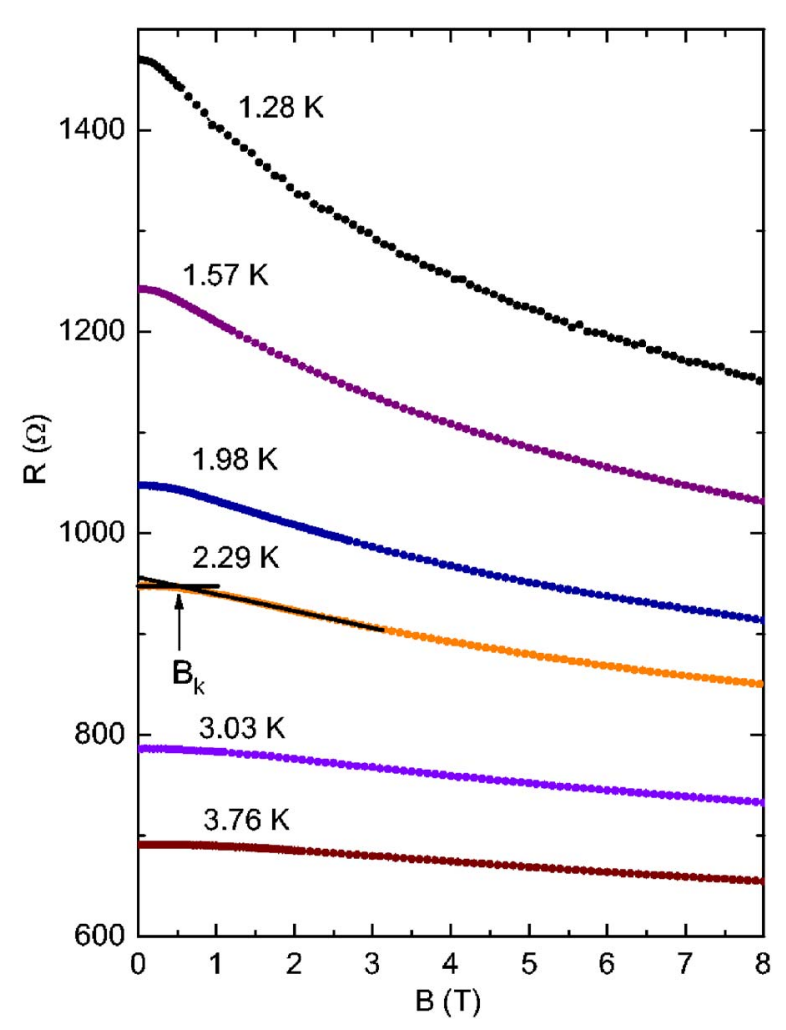

FIG. 2. (Color online) Magnetic field dependence of the van der Pauw resistance for the UNCD sample at different temperatures. Method of determination of the crossover $B_{k}$ between electric and magnetic contributions to the Aharonov-Bohm's phase-breaking process is demonstrated for the $2.29 \mathrm{~K}$ curve.

The influence of relatively weak electromagnetic fields on the sample resistance, encountered in our experiments, can be fully accounted for by the phase breaking process, treated within the frame of Aharonov-Bohm's theory. ${ }^{11}$ Accordingly, the change of the phase $\Delta \varphi$ of a partial electron wave along a path $C$ connecting scattering centers in the cluster is given by an integral

$$
\Delta \varphi=\frac{e}{\hbar} \int_{C}\left(\frac{\partial t}{\partial \mathbf{x}} \psi-\mathbf{A}\right) d \mathbf{x}
$$

where $\psi$ represents the time dependent electric scalar potential and $\mathbf{A}$ the space dependent magnetic vector potential. It is obvious that if the phase of the partial waves is changed appreciably, (we use for the sake of definiteness $\Delta \varphi \approx 1$ ), the stable interference pattern can no longer exist, the electron is released from weakly localized orbit and starts to contribute to the conductivity of the sample. It is apparent from formula (2) that the phase breaking Aharonov-Bohm's process behind may have two independent origins corresponding to electric and magnetic terms, respectively. Since the electromagnetic oscillations (e.g., optical phonons) in the sample at finite temperatures inevitably persist, the electron phase breaking due to the electric term must always be effective there. At zero magnetic fields, it is just this electric-term induced process which by limiting the maximal extent of weakly localized orbits $\approx L_{\varphi}(0)$ preserves a part of charge carriers free, and, in this way controls the conductivity of the sample. As the number of free carriers in the system is proportional to the number of virtual orbits the dimensions of which exceed $L_{\varphi}(0)$, the corresponding enhancement of conductance $\left(G-G_{0}\right)$, usually called "quantum correction," can be tenta- 


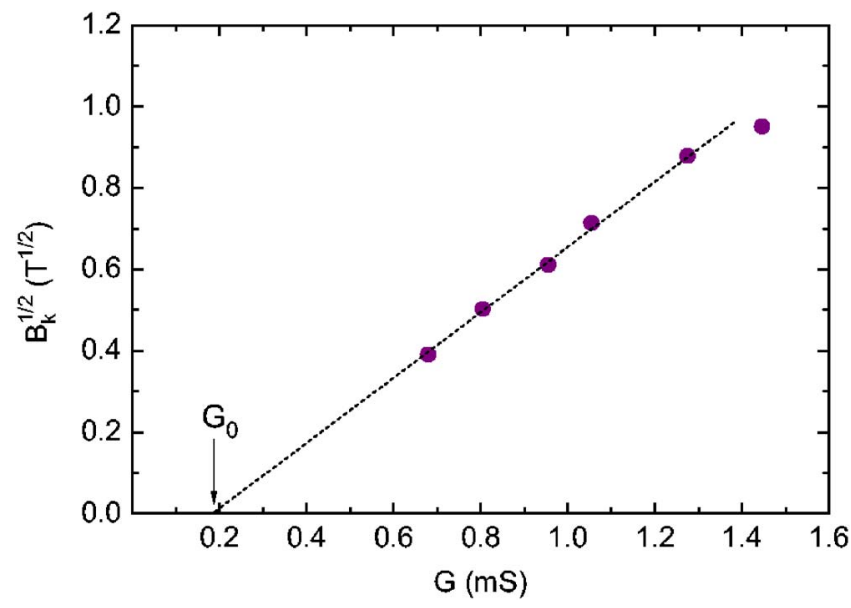

FIG. 3. (Color online) Correlation between the square-roots of the critical magnetic fields $B_{k}$ and zero- $B$ values of conductance $G$ taken at various temperatures. Straight regression line demonstrates the linearity of the correlation as is required by Eq. (6). Its slope and intercept with the $G$ axis provide the lacking parameters $\gamma \approx 3.2 \times 10^{10} \Omega / \mathrm{m}$ and $G_{0} \approx 1.9 \times 10^{-4} \mathrm{~S}$.

tively expressed by a simple relation (cf. Ref. 12)

$$
\gamma\left(G-G_{0}\right)=1 / L_{\varphi}(0),
$$

where $\gamma$ and $G_{0}$ are empirical constants. The relative significance of electrically and magnetically induced AharonovBohm's phase breaking processes on the transport in UNCD film may be assessed as follows. Applying Stokes's formula to the magnetic term in Eq. (2), one can immediately reformulate a condition for the on-set of magnetically induced phase-breaking sufficient for the destruction of interference pattern $(\Delta \varphi \approx 1)$ in terms of magnetic field $|\mathbf{B}|=B$. Thus, the complete phase-breaking should take place if the dimensions of the weak localization (WL) orbit exceed the so called magnetic length

$$
\lambda=(\hbar / e B)^{1 / 2} \text {. }
$$

Consequently, the external magnetic fields delocalize (i.e., destroy) the orbits the extent of which, already limited by oscillating electric field to the value $\sim L_{\varphi}(0)$, is larger than magnetic length $\lambda$. The magnetic field $B_{k}$ at which the contributions of the electric and magnetic terms to the phasebreaking process are just the same is then given by

$$
L_{\varphi}(0)=\left(\hbar / e B_{k}\right)^{1 / 2} \text {. }
$$

In the experimental plot (see Fig. 2) $B_{k}$ can be geometrically constructed as an intersection of a parallel to the $B$ axis, passing through a point corresponding to the resistance at $B=0$ with an extrapolation of the straight line fitting to the linearly decreasing part of the curve at higher fields. Eliminating from Eqs. (3) and (5) the microphysical quantity $L_{\varphi}(0)$, the following correlation accessible to the direct experimental check should be expected:

$$
\sqrt{B_{k}}=\gamma(\hbar / e)^{1 / 2}\left(G-G_{0}\right) .
$$

Actually, such a correlation does exist as is shown in Fig. 3, where $\sqrt{B_{k}}$ versus $G$ is plotted. This plot enables one to determine the constants $G_{0}$ and $\gamma$ and, moreover, approving experimentally relation (6) it confirms indirectly also the validity of Eqs. (3) and (5). The latter equation may then provide a reasonable estimate for the dimensions of the Weakly localized orbits involved. For example, at d.28 K where $B_{k}=0.153 \mathrm{~T}$ we immediately obtain that $L_{\varphi}(0) \approx 6.6$ $\times 10^{-8} \mathrm{~m}$. Comparing this figure with transmission electron microscopy data, we can see that the extent of phasecoherent wave functions is comparable with the grain size $\left[\approx 16 \mathrm{~nm} \approx L_{\varphi}(0) / 2 \pi\right]$. Since the WL effect in threedimensional systems is known to be very small, ${ }^{10}$ the observed giant negative magnetoresistance has to be related to the transport through a system with topological dimension $<3$. Taking into account also the conjecture presented, e.g., in Ref. 13, the only confined subsystem in N-doped UNCD having such properties seems to be that formed at grain boundaries. The existence of the giant NMR observed in our samples excludes simultaneously the possibility of interpreting the transport mechanism in terms of the previously suggested hopping between strongly localized sites (cf. Ref. 6). Indeed, in such a case the presence of a magnetic field would bring about the shrinkage of localized wave functions to the extent $\leqslant \lambda$ [see formula (4)]. It would give further rise, because of the reduction of overlap integrals between the relevant sites, to a large positive magnetoresistance, in a sharp contrast with the experimental data presented in this letter.

Summarizing, we have experimentally studied the lowtemperature magnetotransport properties of highly nitrogen doped ultrananocrystalline diamond films. At temperatures between 90 and $440 \mathrm{~K}$ the samples were proved to be weakly sensitive to external magnetic fields, having relatively high conductivity $\left(\approx 4200 \mathrm{~S} \mathrm{~m}^{-1}\right.$ at room temperature) which was only slightly dependent on temperature $\left(E_{A}<k T\right)$. At temperatures ranging from 1.28 to $3.8 \mathrm{~K}$ the giant negative magnetoresistance reaching up to $22 \%$ at $8 \mathrm{~T}$ was observed. The analysis of experimental data provides the evidence that the transport in these samples is confined to the grain boundaries. The conductance of this subsystem, which has a character of low-dimensional disordered metal, is controlled by the capture of carriers on WL orbits, a typical extent of which is comparable with the grain size $(\approx 16 \mathrm{~nm})$.

The work was partially supported by the Grant Agency of ASCR Contract No. A1010404 and by the Czech Science Foundation Contract Nos. 202/03/0410 and 202/06/0040 and by Institutional Research Plan No. AV0Z10100521.

${ }^{1}$ D. M. Gruen, Annu. Rev. Mater. Sci. 29, 211 (1999).

${ }^{2}$ S. Bhattacharyya, O. Auciello, J. Birrell, J. A. Carlisle, L. A. Curtiss, A. N. Goyette, D. M. Gruen, A. R. Krauss, J. Schlueter, A. Sumant, and P. Zapol, Appl. Phys. Lett. 79, 1441 (2001).

${ }^{3}$ O. A. Williams, S. Curat, J. Gerbi, D. M. Gruen, and R. B. Jackman, Appl. Phys. Lett. 85, 1680 (2004).

${ }^{4}$ A. Härtl, E. Schmich, J. A. Garrido, J. Hernando, S. C. R. Catharino, S. Walter, P. Feulner, A. Kromka, D. Steinmüller, and M. Stutzmann, Nat. Mater. 3, 668 (2004).

${ }^{5}$ M. Nesládek, Semicond. Sci. Technol. 20, R19 (2005).

${ }^{6}$ S. Bhattacharyya, Phys. Rev. B 70, 125412 (2004).

${ }^{7}$ Y. Dai, D. Dai, C. Yan, B. Huang, and S. Han, Phys. Rev. B 71, 075421 (2005).

${ }^{8}$ J. J. Mareš, P. Hubík, J. Krištofik, B. Štěpánek, V. Šestáková, and L. Pekárek, Mater. Sci. Eng., B 28, 134 (1994).

${ }^{9}$ G. Bergmann, Phys. Rev. B 28, 2914 (1983).

${ }^{10} \mathrm{H}$. Fukuyama, in Electron-Electron Interactions in Disordered Systems, edited by A. L. Efros and M. Pollak (North-Holland, Amsterdam, 1985), p. 155.

${ }^{11}$ Y. Aharonov and D. Bohm, Phys. Rev. 115, 485 (1959).

${ }^{12}$ J. J. Mareš, J. Krištofik, and P. Hubík, Solid State Commun. 101, 243 (1997).

${ }^{13}$ P. Zapol, M. Sternberg, L. A. Curtiss, T. Frauenheim, and D. M. Gruen, Phys. Rev :B 65: 0454.03 (2001). aip. org/termsconditions. Downloaded to IP: 